\title{
¿LA VACUNA BCG PUEDE EVITAR EL CÁNCER DE VEJIGA?
}

\author{
E.A. GRANADOS LOARCA, V.L. AMBROSIO \\ Servicio de Urología. Torre profesional, Quetzaltenango. Hospital Regional de Occidente. \\ Quetzaltenango. Guatemala.
}

Actas Urol Esp. 28 (5): 350-353, 2004

\section{RESUMEN}

¿LA VACUNA BCG PUEDE EVITAR EL CÁNCER DE VEJIGA?

En los países en vía de desarrollo la incidencia de tuberculosis es alta, y la incidencia de cáncer de vejiga es baja. Sobre la base de la utilidad del BCG en la prevención de la TBC y de su uso en el tratamiento y de prevención de las recurrencias del cáncer de vejiga, se investigó la relación entre las 2 patologías.

MATERIAL Y MÉTODOS: Se efectuó un estudio retrospectivo y prospectivo. Se revisaron las historias de 52 casos de cáncer de vejiga, se entrevistaron 100 personas, y se entrevistó a médicos que se dedican a tratar pacientes con tuberculosis, sobre la existencia de algún paciente que hubiese tenido las 2 patologías o de algún caso vacunado con BCG, que hubiera desarrollado cáncer de vejiga. Éste es un estudio hecho en tres grupos de pacientes, en un periodo de 14 años, entre 1985 y 1999.

RESULTADOS: Primer grupo: todos los casos que desarrollaron cáncer de vejiga a ninguno se le había administrado la vacuna de BCG. Segundo grupo: todos los que recibieron la vacuna de BCG como prevención de TBC pulmonar, ninguno desarrollo cáncer de vejiga en el transcurso de una media de 32 años. Tercer grupo: ningún paciente que fue tratado por tuberculosis desarrolló cáncer de vejiga. Ninguno de los médicos entrevistados habían observado caso alguno que hubiera desarrollado las dos patologías, o pacientes que se hubieran vacunado con BCG, que desarrollara cáncer de vejiga.

PALABRAS CLAVE: Cáncer de vejiga. BCG. Prevención.

\section{ABSTRACT \\ COULD THE BCG VACCINE PREVENT BLADDER CANCER?}

In developed countries, the incidence of tuberculous is major, and the incidence of bladder cancer is minor. Above the base of the utility of the BCG in the prevention of the tuberculous and the use in the treatment and prevention of the recurrences of cancer, it's investigated the relation between the 2 pathology's.

PATIENTS AND METHODS: We effected a study retrospective and prospective. It's revised the history's of 52 cases of bladder cancer, it's interview 100 persons, and interview medicals what to dedicate to treat patients whit tuberculous, above the existence before patient what had the 2 pathology's or before case vaccinate with BCG what to develop bladder cancer. It's a study effected in tree groups of patients, in a period of 14 year, between 1985-1999.

RESULTS: First group: all the cases what development bladder of cancer none of them to vaccinate with BCG. Second group: all to vaccinate with BCG with prevention of lung tuberculous none of them development bladder of cancer in the course of mean of 32 year. Third group: none of the patients what to treat for tuberculous developed bladder of cancer. Never a medical interview observe case some what development the two pathology's, or patients what vaccinate with BCG what development bladder of cancer.

KEY WORDS: Bladder cancer. BCG. Prevention. 
$\mathrm{L}^{2}$ a alta incidencia de tuberculosis (TBC), y la baja incidencia de cáncer vesical en los países en vías de desarrollo. El aumento del número de personas vacunadas en nuestro país con BCG para prevenir la tuberculosis pulmonar. La utilización de la BCG en la prevención de recurrencias y en el tratamiento del cáncer de vejiga en los países desarrollados, nos motivó a que efectuáramos este estudio.

\section{MATERIAL Y MÉTODOS}

En los países en vía de desarrollo la incidencia de tuberculosis es alta, y la incidencia de cáncer de vejiga es baja. Sobre la base de la utilidad del BCG en la prevención de la TBC y de su uso en el tratamiento y de prevención de las recurrencias del cáncer de vejiga, se investigó la relación entre las 2 patologías. Para ello se efectuó un estudio retrospectivo y prospectivo. Se revisaron las historias de 52 casos de cáncer de vejiga, se entrevistaron 100 personas, y se entrevisto a médicos que se dedican a tratar pacientes con tuberculosis, sobre la existencia de algún paciente que hubiese tenido las 2 patologías o de algún caso vacunado con BCG que hubiera desarrollado cáncer de vejiga. Este es un estudio hecho en tres grupos de pacientes, en un periodo de 14 años, entre 1985 y 1999.

Primer grupo: 52 casos de cáncer de células transicionales de vejiga en una población de 500.000 habitantes, 36 hombres y 16 mujeres (3,7 casos $x$ año). La edad osciló entre 40-68 años (media 53). 3 casos en estadio A, 8 estadio B, 12 estadio C, 18 estadio D1, 11 estadio D2. (Jewett y Marshall). 15/52 pacientes eran fumadores (Tabla I).

Segundo grupo: 100 pacientes, 50 hombres y 50 mujeres. La edad osciló entre 40-70 años (media de 55), a quienes se les había administrado previamente la vacuna del BCG, 10 unidades de BCG Pasteur, para prevenir la tuberculosis pulmonar, entre 28-55 años antes (media de 32).

Tercer grupo: 100 pacientes, 50 hombres y 50 mujeres. La edad osciló entre 40-65 años (media de 52), que recibieron tratamiento por TBC pulmonar.

A los tres grupos se les entrevistó y se mantuvo comunicación con la mayor parte de ellos.
TABLA I

DATOS DE LOS PACIENTES CON CÁNCER DE VEJIGA

\begin{tabular}{|c|c|}
\hline Epidemiologia & No \\
\hline Sexo/Raza & \\
\hline Hombres blancos & 10 \\
\hline Hombres negros & 2 \\
\hline Hombres indígenas & 24 \\
\hline Mujeres blancas & 8 \\
\hline Mujeres negras & 0 \\
\hline Mujeres indígenas & 8 \\
\hline Edad diagnóstico & 40 a 68 años \\
\hline Área rural & 31 \\
\hline Área urbana & 21 \\
\hline Factores de Exposición Ocupacional & \\
\hline Insecticidas y abonos (agricultores) & 18 \\
\hline Comerciantes & 7 \\
\hline Impresores & 1 \\
\hline Oficinistas & 11 \\
\hline Barbero & 1 \\
\hline Oficios domésticos & 6 \\
\hline Cocineras & 8 \\
\hline \multicolumn{2}{|l|}{ Fumadores cigarrillos } \\
\hline$<5$ cigarrillos/día & 5 \\
\hline$>5$ cigarrillos/día & 10 \\
\hline \multicolumn{2}{|l|}{ bebedores de café o té } \\
\hline 1-2 tazas/día & 6 \\
\hline$>3$ tazas/día & 18 \\
\hline Analgésicos (acetominofen) & 11 \\
\hline Infecciones urinarias & 16 \\
\hline Litiasis vesical & 9 \\
\hline \multicolumn{2}{|l|}{ Tipos de sangre } \\
\hline $\mathrm{O}+$ & 28 \\
\hline $\mathrm{A}+$ & 19 \\
\hline $\mathrm{B}+$ & 4 \\
\hline $\mathrm{AB}+$ & 1 \\
\hline
\end{tabular}

\section{RESULTADOS}

Primer grupo: todos los casos que desarrollaron cáncer de vejiga, a ninguno se le había administrado la vacuna de BCG.

Segundo grupo: todos los que recibieron la vacuna de BCG como prevención de TBC pulmonar, ninguno desarrolló cáncer de vejiga en el transcurso de una media de 32 años.

Tercer grupo: ningún paciente que fue tratado por tuberculosis desarrolló cáncer de vejiga. 
Ninguno de los médicos entrevistados habían observado caso alguno que hubiera desarrollado las dos patologías, o pacientes que se hubieran vacunado con BCG que desarrollara cáncer de vejiga.

En nuestro país en vía de desarrollo, el cáncer de vejiga es poco frecuente, es 2,2 veces más común en hombres que en mujeres. El promedio de edad es 53 años, bajo en comparación con otras series. El $78 \%$ de los pacientes con cáncer de vejiga diagnosticados estaban en un estadio C.

\section{DISCUSIÓN}

La organización mundial de la salud ha estimado el aparecimiento en el mundo de 10 millones de nuevos casos de todas las formas de tuberculosis cada año, su mayor parte en países del tercer mundo, donde continúa siendo un problema mayor.

Calmette y Guérin desarrollan una vacuna para prevenir la tuberculosis, y consiguen después de 13 años un bacilo tuberculoso atenuado, no lesivo pero sí antigénico, con dos efectos fundamentales: 1) Como prevención de la tuberculosis; y 2) Estimulante. Los dos métodos básicos para planificar eficientemente un control de la tuberculosis son: 1) La inoculación de la vacuna Bacilo Calmette-Guérin (BCG); y 2) El tratamiento temprano.

En los países en desarrollo, la inmunización con la vacuna BCG juega un papel muy importante en el control de esta enfermedad, si ésta se organiza de una manera eficaz administrando la vacuna después del nacimiento, a pesar de que se ha sugerido que la vacuna BCG no previene la infección sino que limita la diseminación y multiplicación de M. Tuberculosis.

El cáncer de vejiga es más frecuente en países desarrollados que en países en vías de desarrollo. $\mathrm{Su}$ incidencia y su mortalidad son cada vez mayores.

Aproximadamente el 70\% de los cánceres vesicales son de grado bajo, y superficiales ${ }^{1}$. Es en estos pacientes donde el tratamiento del BCG está indicado, ya que mejoran o se curan con su administración ${ }^{2}$. Estudios a largo plazo con terapia de BCG han reportado respuestas favorables $^{3,4}$.
La BCG intravesical ${ }^{2}$, se administra en cuatro situaciones clínicas:

1. Como profilaxis en pacientes con cáncer vesical.

2. En el tratamiento del tumor residual, en pacientes con cáncer de células transicionales, y papilares.

3. En el tratamiento de tumores de alto grado.

4. En el tratamiento de pacientes con carcinoma in $\mathrm{situ}^{3,6}$. La BCG es una muestra atenuada del Mycobacterium Bovis que tiene efectos estimulatorios y respuestas inmune ${ }^{3-5}$.

La terapia con BCG se ha sugerido que tiene efectos antitumorales a través de mecanismos inmunes ${ }^{6}$. La BCG se ha utilizado en la prevención de cualquier tipo de tuberculosis, es un virus atenuado que se administra por vía intradérmica. La BCG ha sido bien usada en la prevención y tratamiento del cáncer de vejiga. La vía que se utiliza es la intravesical, aunque se han utilizado otras vías como la intradérmica o la oral, sin resultados satisfactorios. Los pacientes que han desarrollado tuberculosis han tenido el bacilo vivo, el mismo bacilo vivo o atenuado puede dar la inmunidad contra el cáncer de vejiga.

Pearl en 1929 descubrió que los pacientes que habían sufrido de tuberculosis, tenían menor incidencia de cáncer en general.

Para que el BCG prevenga la tuberculosis, posiblemente el cáncer de vejiga se tendría que administrar 30-40 años antes de la edad promedio en la cual se desarrolla la enfermedad, es decir a los 12-15 años de edad.

Para concluir, la administración de BCG en los pacientes con cáncer de vejiga superficial es de utilidad en la prevención y tratamiento. La administración de la vacuna BCG previene la tuberculosis. Y cuestiono ¿la tuberculosis da inmunidad contra el cáncer de vejiga?, ¿puede la vacuna BCG disminuir la incidencia del cáncer de células transicionales de vejiga?

\section{REFERENCIAS}

1. CATALONA WJ, RETLIFF TL.: Bacilus CalmetteGuérin and superficial bladder cancer: clinical experience and mechanism of action. Surg Annu 1990; 22: 363 . 
2. MORALES A, EIDINGER D, BRUCE AW.: Intracavitary bacillus Calmette-Guérin in the treatment of superficial bladder tumors. J Urol 1976; 116: 180 .

3. HERR HW.: Progression of stage T1 bladder tumors after intravesical bacillus Calmette-Guérin. J Urol 1991; 145: 40-44.

4. HILLYARD RW, LADAGA L, SCHELLHAMMER PF.: Superficial transitional cell carcinoma of the bladder associated with mucosal involvement of the prostatic urethra: results of treatment with intravesical bacillus Calmette-Guérin. J Urol 1988; 139: 290293.

5. BROSMAN SA, LAMM DL.: The preparation, handling and use of intravesical bacillus Calmette-Guérin for management of stage Ta, T1, carcinoma in situ and transitional cell cancer. J Urol 1990; 144: 313315.

6. DROLLER MJ.: Bacillus Calmette-Guérin in the management of bladder cancer. J Urol 1986; 135: 331-333.

Dr. E.A. Granados

31 Avda "F" 14-60 Zona 7

Cond. Villas de San Martín

Guatemala

(Trabajo recibido el 7 mayo de 2003) 